\title{
Review \\ Artificial Intelligence Computing at the Quantum Level
}

\author{
Olawale Ayoade ${ }^{1}\left[\right.$, Pablo Rivas ${ }^{2, *}(\mathbb{C})$ and Javier Orduz ${ }^{2} \mathbb{C}$ \\ 1 Department of Physics, Baylor University, One Bear Place \#97316, Waco, TX 76706, USA; \\ olawale_ayoade1@baylor.edu \\ 2 Department of Computer Science, Baylor University, One Bear Place \#97141, Waco, TX 76712, USA; \\ javier_orduzducuara@baylor.edu \\ * Correspondence: pablo_rivas@baylor.edu
}

Citation: Ayoade, O.; Rivas, P.; Orduz, J. Artificial Intelligence Computing at the Quantum Level. Data 2022, 7, 28. https://doi.org/ $10.3390 /$ data7030028

Academic Editors: Pufeng Du and Jialin Zhang

Received: 30 December 2021 Accepted: 21 February 2022 Published: 25 February 2022

Publisher's Note: MDPI stays neutral with regard to jurisdictional claims in published maps and institutional affiliations.

Copyright: (C) 2022 by the authors. Licensee MDPI, Basel, Switzerland. This article is an open access article distributed under the terms and conditions of the Creative Commons Attribution (CC BY) license (https:// creativecommons.org/licenses/by/ $4.0 /)$.

\begin{abstract}
The extraordinary advance in quantum computation leads us to believe that, in the not-toodistant future, quantum systems will surpass classical systems. Moreover, the field's rapid growth has resulted in the development of many critical tools, including programmable machines (quantum computers) that execute quantum algorithms and the burgeoning field of quantum machine learning, which investigates the possibility of faster computation than traditional machine learning. In this paper, we provide a thorough examination of quantum computing from the perspective of a physicist. The purpose is to give laypeople and scientists a broad but in-depth understanding of the area. We also recommend charts that summarize the field's diversions to put the whole field into context.
\end{abstract}

Keywords: quantum computing; quantum artificial intelligence; quantum information

\section{Introduction}

Quantum computing (QC) is gaining popularity at a rapid pace. Its adaptability is attracting enough attention to help it grow. Top enterprises from around the world, research institutions, startups, and organizations with sufficient resources have all contributed to the advancement of this remarkable sector. While many are skeptical of its ability to achieve exceptional results at the level it claims, others are excited about the solutions it can provide to modern-day challenges that are beyond the reach of traditional classical computing (CC). It is vital to note that $\mathrm{QC}$ is not a technique or a paradigm for subduing or suppressing CC in and of itself, but rather for picking up the pace where CC may be behind.

Quantum machine learning (QML), a growing topic that blends quantum information (algorithms) with machine learning (ML) [1], or classical machine learning algorithms applied on quantum devices, is one of the models in QC. We hope to tackle problems with improved performance using this blending region, and in terms of complexity theory, we should be able to reduce the running time and memory space.

On a quantum computer, quantum algorithms are step-by-step operations [2]: these operations, which use quantum mechanics concepts, such as superposition and entanglement, improve speed, optimization, and other efficient computations that cannot be performed on a traditional computer. Although this is a developing topic, much work has been done to design algorithms that will function with quantum devices in the near future. Ref. [3] provides a complete list of accessible quantum algorithms.

\subsection{Contributions}

The following are the specific contributions of our paper:

1. This paper provides foundational concepts about QC and a comprehensive analysis of recent work, investigating the connection between physics and information science and assessing the area's growth.

2. We present flowcharts that summarize the field's development and/or roadmap, as well as a comparison of common quantum bit technologies and open source development frameworks. 
3. We explore the field's current (experimental and theoretical) problems in achieving fully scaled quantum devices. We also provide a summary of past developments as well as a progress report on quantum information science topics of interest.

4. We provide a list of topics of interest, together with a high-quality list of reference resources for a more in-depth study of each subject, based on the preferences of the readers.

\subsection{Structure}

We begin by providing a layman introduction to quantum physics and machine learning, followed by a concise overview of quantum information science related to those subjects. We then present a complete list of effective QC models, along with research developments. As indicated in several articles, we propose various implementable concepts or future work. Finally, we look at a variety of cutting-edge applications that are (or have been) already implemented, as well as how we perceive QC will be used in the near future.

In the following section, this paper explores topics related to modern physics and artificial intelligence. After that, we go over some quantum information ideas. We devote a part to providing a quantum computing timeline, followed by a discussion and conclusion.

\section{Modern Physics and Artificial Intelligence}

This part begins with a brief introduction to quantum mechanics, followed by a discussion of machine learning. The next section explains how these two sciences were joined with other fields of information science to establish quantum computing.

\subsection{Quantum Mechanics}

Quantum mechanics is the foundational theory that explains and provides all knowledge about matter and light's behavior. Objects in quantum physics have wave-like qualities [4]. Despite the prevalent concept that quantum mechanics is limited to subatomic particles, predictions have been made that QM will solve computational difficulties in domains such as chemistry, physics, machine learning, and communication system security enhancement [5].

\section{Quantum Theory}

Quantum mechanics is a framework for understanding quantum phenomena [4]. This framework provides information on a particle's state described by a wave function, commonly represented as $\psi(x, t)$. The Schrödinger equation describes the time evolution of this wavefunction, which contains all available information about the particle [6]:

$$
i \hbar \frac{\partial|\psi(t)\rangle}{\partial t}=\hat{H}(t)|\psi(t)\rangle \text {. }
$$

where $\hbar$ is Planck's constant and $\hat{H}(t)$ is the Hamiltonian operator, which, for general purposes, represents the energy of the system.

\subsection{Machine Learning}

ML was coined by Arthur Samuel in 1959 to describe the field in which computers may learn to do tasks without being instructed to do so [7,8]. Rivas [9] gave a depiction of the ML ecosystem, while [8] depicted the multi-disciplinary fields of ML. The goal of machine learning is to create algorithms that can learn from data on their own [10].

ML can be supervised or unsupervised [11]. Unsupervised learning is a technique to learning from data, where multiple supervisory signals self-optimize a fitness function. In contrast, supervised learning is a process where the algorithm learns from labels as supervisory signals [9]. In recent years, ML has developed rapidly to higher dimensions, such as deep learning, whose applications such as image classification, autonomous car driving, speech recognition, and more are applicable to everyday life [12]. 
Classical information theory, computer science, and quantum physics are all combined in quantum computing. As a result, we can conclude that quantum information, which has three main areas: quantum computing, quantum information theory, and quantum cryptography. We shall discuss about the quantum computing, namely, the area of inquiry that uses quantum phenomena, such as interference, superposition, and entanglement, and Dirac or bracket notation; to operate on quantum states, these states represent data [13].

\section{Quantum Information Science}

As previously remarked, quantum computing is coined from two areas of sciences: physics (quantum mechanics) and information science (mathematics, theoretical and applied computer science, among others).

The dots between information science and quantum physics principles are connected here. We begin by outlining the postulates of quantum mechanics as they relate to quantum information theory. We also include a one-line statement that defines quantum information processing terminologies, such as entanglement, superposition, and speedup, among others. This summary is based on Refs. [1,13-18].

\subsection{Quantum Mechanics Postulates, Entanglement, Mixed States and Operations}

The unique feature of a quantum computer in comparison to a classical computer is that the bit (often referred to as "qubit") can be in one of two states ( 0 or 1 ) and possibly a superposition of the two states (a linear combination of 0 and 1) per time. The most common mathematical representation of a qubit is

$$
|\psi\rangle=\alpha|0\rangle+\beta|1\rangle
$$

Equation (2) is a superposition state, where $\alpha, \beta$ are complex numbers and $|0\rangle,|1\rangle$ are computational basis states that form an orthonormal basis in this vector space. A qubit can also be represented visually using a Bloch sphere, as shown in Figure 1.

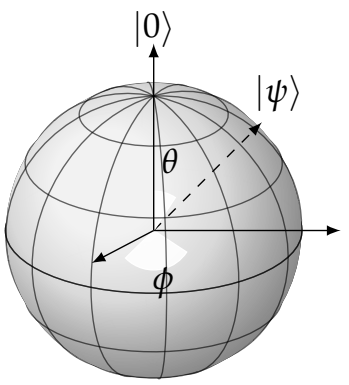

$|1\rangle$

Figure 1. A Bloch sphere. This is used to visualize the qubit's geometric state. It gives the angles and basis vectors for the $|\psi\rangle$ representation.

We then move on to the postulates of quantum mechanics, which are preceded by a diagram in Figure 2 that gives a summary representation of the postulates.

- $\quad$ Postulate 1: State Space. The state of a quantum system is described by a unit vector $|\psi\rangle$ that lives in a Hilbert space $\mathcal{H}$. This state contains all necessary information to characterize the system.

- $\quad$ Postulate 2: Evolution. A closed quantum system undergoes a time evolution $|\psi(t)\rangle$. This evolution is described by a unitary transformation that follows the Schrodinger Equation (1).

- $\quad$ Postulate 3: Measurement. Quantum measurements can be expressed using sets of measurement operators $\left\{M_{m}\right\}$. In an experiment, $m$ represents the possible measurement outcomes. Upon measuring a state, say $|\psi(t)\rangle$, the probability of an $m$ outcome is $p(m)$. 
- $\quad$ Postulate 4: Composite Systems. Two or more physical systems can be treated as a composite system. The state space of a composite system is the tensor product space of the states of the component physical systems.

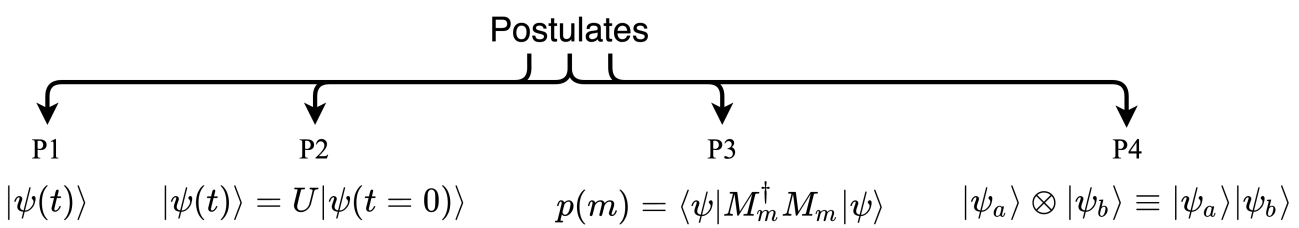

Figure 2. One easy way to show the quantum mechanics postulates chart. P1 defines the state space, P2 defines the evolution, P3 defines the measurement, and P4 defines the composite system, where $\left|\psi_{a}\right\rangle\left|\psi_{b}\right\rangle \equiv\left|\psi_{a} \psi_{b}\right\rangle$.

Other Important Properties

- $\quad$ Superposition Linear combination of two states.

- Entanglement When the values of specific qualities of one system are correlated with the values of the corresponding properties of the other system, two quantum systems are said to be entangled.

- $\quad$ Speedup If the quantum algorithm requires fewer queries to solve a problem than the classical approach, the outcome is a quantum speedup [19].

\subsection{Quantum Information Science: An Overview}

Quantum communication, quantum computing, and quantum sensing (and metrology) are the three research categories in quantum information science. There are subcategories and specific research activities that pertain to each of these groups-starting with quantum communication, which is essentially the process of exchanging information on a quantum level. Quantum cryptography and networking are two popular uses of this burgeoning field. Moving on to quantum sensing, this branch of quantum information studies how to make quantum devices interact with their surroundings. Quantum systems and designs are examples of this type of application. In the subject of quantum information science, quantum computing is the third most talked-about topic.

Quantum computers are now divided into two types: analog and digital. The analog computer is a quantum computer that runs based on the system's Hamiltonian and the initial quantum state of qubits. Three forms of analog quantum computers exist:

- $\quad$ Adiabatic quantum computing $(A Q C)$ is a computational model that employs adiabatic quantum mechanical processes [20].

- Quantum annealing $(Q A)$ is a technique for evaluating the minimum of an objective function that is built on AQC concepts but does not meet its stringent requirements [20].

- Quantum simulation (QS) is the use of a controllable quantum system to examine a less controllable or accessible quantum system [21].

The digital quantum computer is the second type of quantum computer. It is a quantum device that computes using a limited number of elementary operations, termed gates, on quantum bits (qubits). Gate-based quantum computing is the most common type of digital computer.

- $\quad$ Gate-based quantum computing (GBQC) accepts data and modifies it by a unitary operation, which is expressed as a sequence of gate operations and measurements (i.e., the algorithm) and may be represented by a quantum circuit [22]. Quantum machine learning is the GBQC's driving force.

\subsection{Quantum Computing System}

As shown in Figure 3, there are two kinds of quantum computers: analog and digital quantum computers. Both of these types of computers have some similarities, which are "hardware" and "software" relative to traditional computers. This is depicted in Figure 4: 


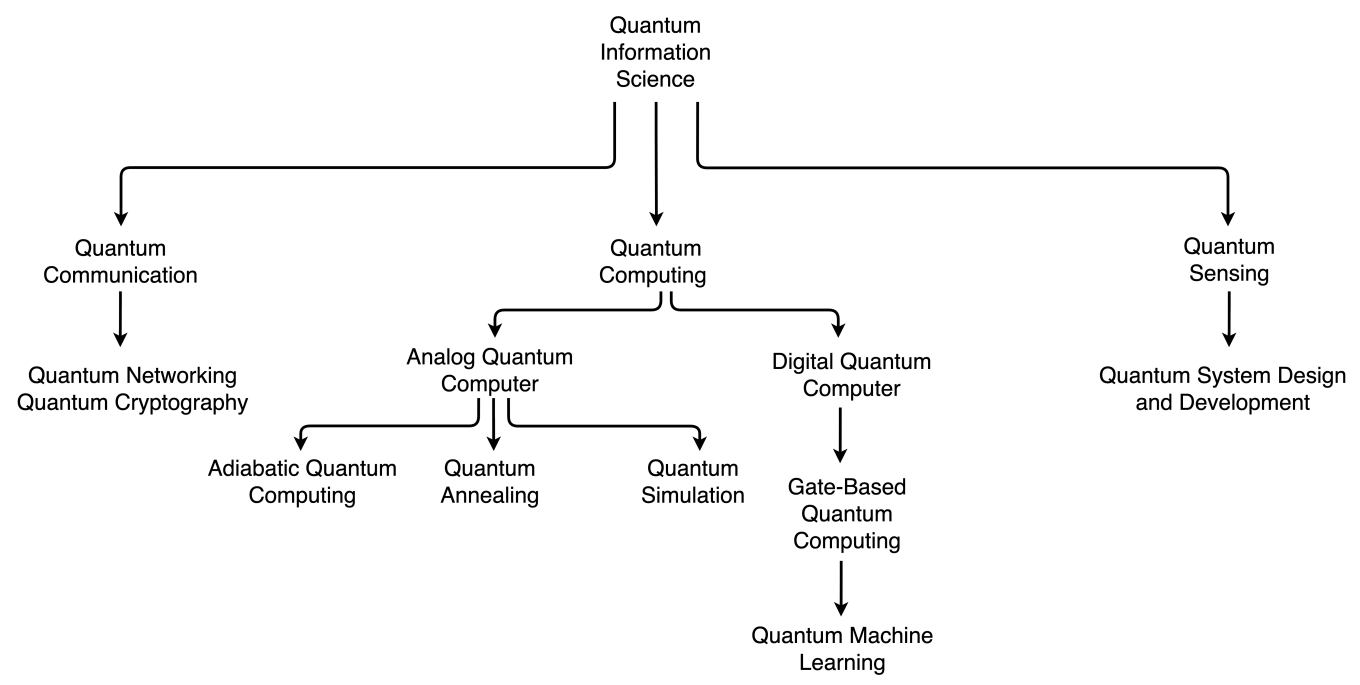

Figure 3. Quantum information science (QIS) chart. QIS is divided into three categories: quantum communication, quantum computing, and quantum sensing (and metrology). Quantum communication and quantum sensing have applications, such as quantum networking and quantum system design, respectively. The field of QC splits into analog and digital computing. An analog quantum computer could be any of three forms: adiabatic quantum computing, quantum annealing, or quantum simulation. A digital quantum computer exists in the form of gate-based quantum computing, using the features of QML for its operations.

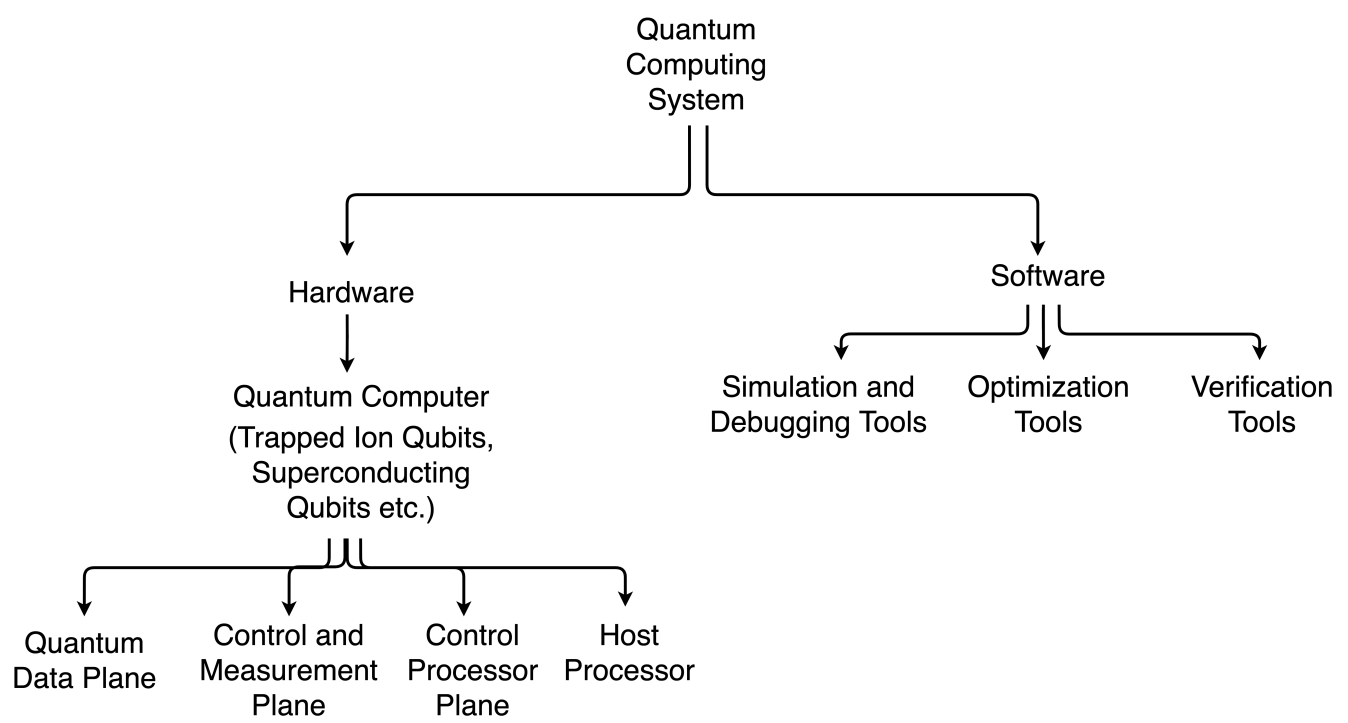

Figure 4. Quantum computing system (QCS) Chart. QCS is a complete system that has two operational parts: hardware and software. The hardware of a QCS is essentially a quantum computer (with variations such as trapped ion qubits and superconducting qubits, among others). Generally, a quantum computer comprises essential components, which are quantum data planes, control and measurement planes, control processor plane, and a host processor. Similarly, the software of a QCS is composed of essential tools, which are simulation and debugging tools, optimization tools, and verification tools.

The hardware of a quantum system, the quantum computer, is made up of various components. The operation of these components necessitates the use of software tools. The various programming tools as they apply to a quantum system are visually represented in Figure 5. 


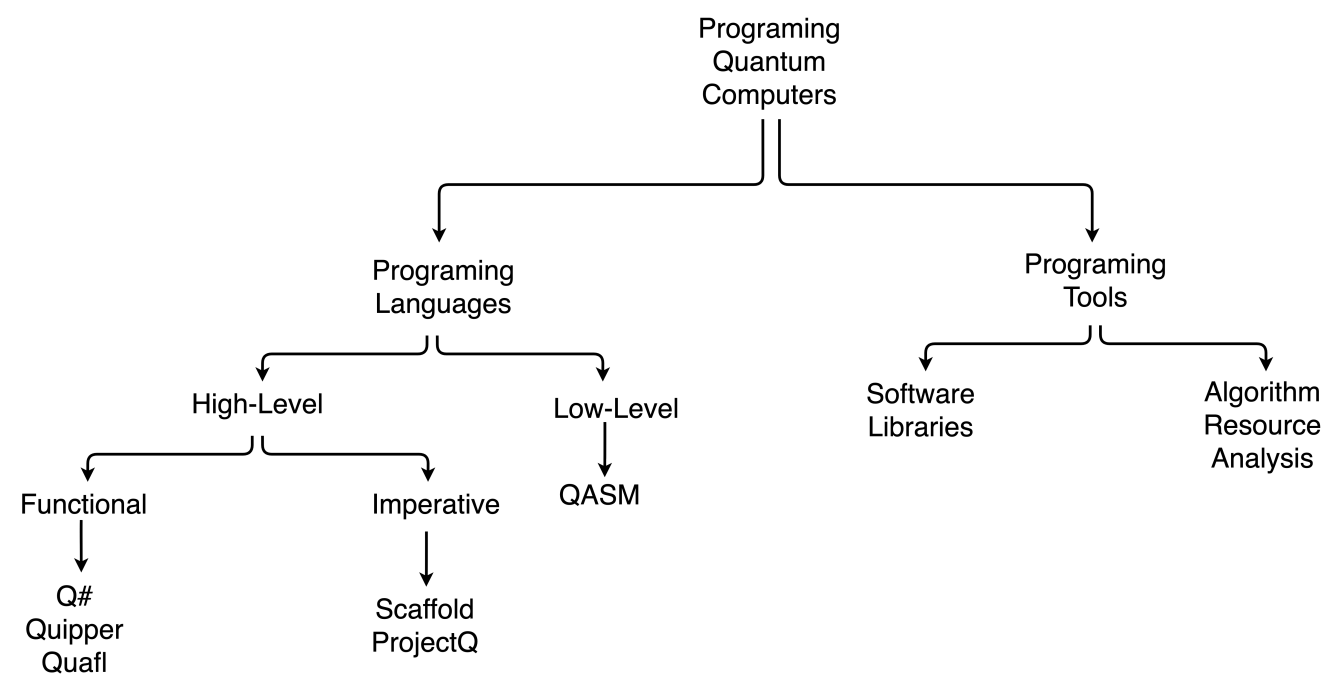

Figure 5. Programming quantum computers Chart. Quantum computers necessitate the use of two key features to run efficiently: programming languages and programming tools. A programming language can either be high level or low level. An example of low-level language programming is quantum assembly language (QASM). A high-level programming language is functional (example includes Quipper) and imperative (example includes ProjectQ).

\subsection{Q-Gates, Circuits, and Algorithms}

A quantum circuit is a representation of a quantum operation that is performed sequentially. Logic qubits are transported on "wires" (shown by horizontal lines), and quantum gates (represented by blocks) act on the qubits in a typical quantum circuit, as depicted in Figure 6. The logical gate is a device that controls or processes data; the Hadamard " $\mathrm{H}$ " gate and NOT " $\mathrm{X}$ " gate are two common examples.

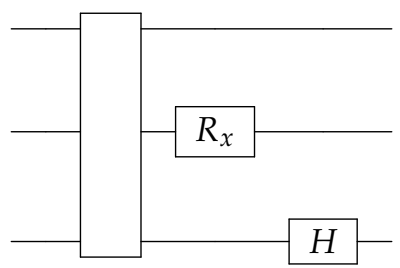

Figure 6. A quantum circuit. The horizontal lines are referred to as wires representing qubits, while the boxes represent operators acting on the qubits. $R_{x}$ and $H$ are the rotation operator and Hadamard gates, respectively.

Algorithms are used in computers to complete predefined tasks. Quantum algorithms work with any actual quantum computation model [23]. Quantum computers can run classical algorithms, but not the other way around. This is due to entanglement and situations such as qubit superposition. Quantum algorithms are created using two key quantum gates-Hadamard and phase gates-which take advantage of the quantum interference, parallelism, and function evaluation properties. The Quantum Algorithm Zoo website [3] includes a thorough list.

In Table 1, we show different quantum algorithms as they fit under various categories of activities and applications. 
Table 1. Quantum algorithm classifications.

\begin{tabular}{ll}
\hline \multicolumn{2}{c}{ Quantum Algorithms } \\
\hline Quantum Fourier transform & Amplitude amplification \\
\hline Simon's algorithm & Grover's algorithm \\
Shor's algorithm & Quantum counting \\
\hline
\end{tabular}

\subsection{Common Quantum Applications}

There are many quantum applications in use today, and we will go over a few of them here: information encoding, quantum teleportation, and quantum cryptography.

\subsubsection{Information Encoding}

Quantum computers are projected to employ quantum data for information processing, similar to how classical computers use classical data for information processing. Because of the established conceptuality of QC outperforming CC, the ability to execute classical operations, and the restrictions in generating quantum data, the problem of converting classical data to quantum data has attracted a lot of attention in recent years, with various advancements. This procedure is known as data encoding.

Because data arrive in various formats, there are multiple methods for transforming conventional data to quantum data. The following is a list of data encoding methods that have been confirmed to work:

- Basis encoding;

- Amplitude encoding;

- Qsample encoding;

- Dynamic encoding.

In [1], a summary is given of the various forms of information encoding that have been described, along with possible outcomes.

\subsubsection{Quantum Teleportation}

Teleportation uses the entanglement property to transport quantum information from one quantum state (qubit) to another. This procedure is frequently used between two parties that are attempting to communicate. There are five phases to transferring an unknown quantum state $q_{0}$ between parties $q_{1} \& q_{2}$ :

1. Parties $q_{1}$ and $q_{2}$ create an entangled pair.

2. $q_{1}$ applies a CNOT gate with the unknown state $q_{0}$.

3. $q_{1}$ applies a Hadamard gate to the first qubit of the result in 2 .

4. $q_{1}$ measures the results from 3 .

5. $q_{1}$ communicates the measurement results with $q_{2}$.

Using an IBM simulator (Qiskit), we give a circuit schematic (Figure 7) of the teleportation process in context.

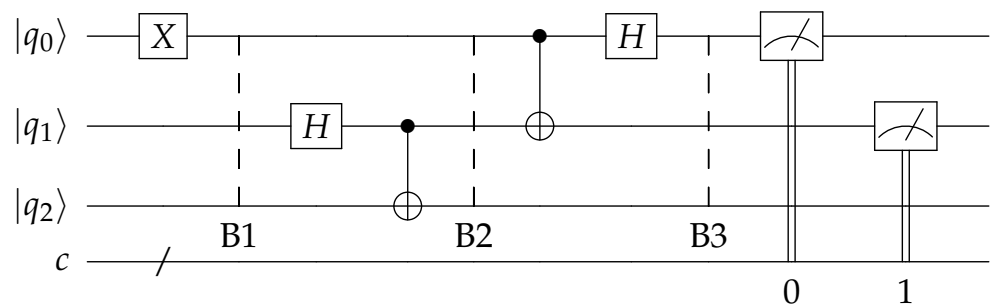

Figure 7. Quantum teleportation circuit. There are three qubits $\left(q_{0}, q_{1}\right.$ and $\left.q_{2}\right)$ and two classical bits (represented by the / sign on the $c$ wire). In addition, there are two Hadamard gates and two CNOT gates. Finally, measurements are made on the classical channels. Each dashed vertical line represents a barrier (B1, B2, and B3) to split some circuit parts for sequential order of operation. 


\subsubsection{Quantum Cryptography}

Quantum key distribution (QKD) is another name for quantum cryptography. QKD is a type of key distribution that encrypts and decrypts communications using quantum physics principles to establish a high-level secure data transmission between two or more parties. As a general rule, today's most popular and commonly utilized form of encryption is known as RSA encryption. Without going into too much detail about RSA because that is outside the scope of this paper, the most important thing to know is that the security system relies heavily on huge numbers that are not impossible to crack but difficult to factor. Peter Shor's prime factorization algorithm (executed on a quantum computer) presents a significant challenge to this method. QKD, which is based on quantum physics, provides a more efficient way of encrypting data.

Principle A secure transmission of QKD necessitates two communication channels. The encrypted message is sent over a classical channel (such as the telephone), while the quantum keys needed to decrypt the message are sent over a quantum channel (such as qubit transfer).

In QKD, numerous protocols are employed. The BB84 (Bennett and Brassard, 1984) protocol was the first to be established, and several protocols have since been built on it. B92, E91, decoy state, and a slew of other protocols are among them.

BB84 This is based on three fundamental aspects of quantum physics:

- No-cloning theorem;

- State collapse when measured;

- Irreversible measurement.

\section{Quantum Computing Frameworks}

In Table 2, we offer a mix of open source quantum computing tools largely driven by large-scale industry players, with the purpose of making it easier to design quantum algorithms.

Table 2. Each quantum computing tool's programming language, computing paradigm, and description are mentioned in the table.

\begin{tabular}{|c|c|c|c|}
\hline Tool & $\begin{array}{l}\text { Programming } \\
\text { Language }\end{array}$ & $\begin{array}{l}\text { Quantum Computing } \\
\text { Paradigm }\end{array}$ & Framework Description \\
\hline Cirq & Python & Discrete gate model & $\begin{array}{l}\text { A library for creating, manipulating, and optimizing } \\
\text { Noisy Intermediate Scale Quantum (NISQ) circuits, } \\
\text { which can then be executed on quantum computers } \\
\text { and simulators. }\end{array}$ \\
\hline dwave-system & Python & Quantum annealing & $\begin{array}{l}\text { An API for using the D-Wave system as a sampler in } \\
\text { the D-Wave Ocean software stack, either directly or } \\
\text { via Leap's cloud-based hybrid solvers. }\end{array}$ \\
\hline FermiLib & Python & Discrete gate model & $\begin{array}{l}\text { An open-source software suite that makes it easier } \\
\text { to create and test algorithms for quantum computer } \\
\text { simulations of fermionic systems. }\end{array}$ \\
\hline Qbsolv & C & Quantum annealing & $\begin{array}{l}\text { A deconstructing solver that splits a huge quadratic } \\
\text { unconstrained binary optimization (QUBO) problem } \\
\text { into pieces to obtain a minimal value. }\end{array}$ \\
\hline QGL.j1 & Julia & Discrete gate model & A QGL compiler with a focus on performance. \\
\hline Qiskit.js & JavaScript & Discrete gate model & Quantum Information Science Kit for JavaScript. \\
\hline Qrack & $\mathrm{C}++$ & Discrete gate model & $\begin{array}{l}\text { A complete framework for constructing universal vir- } \\
\text { tual quantum processors that is GPU accelerated. }\end{array}$ \\
\hline Quirk & JavaScript & Discrete gate model & $\begin{array}{l}\text { A browser-based quantum circuit simulator with } \\
\text { drag-and-drop functionality. A toy for experimenting } \\
\text { with and learning about small quantum circuits. }\end{array}$ \\
\hline $\begin{array}{l}\text { Strawberry } \\
\text { Fields }\end{array}$ & Python & Continuous gate model & $\begin{array}{l}\text { A Python library for developing, optimizing, and } \\
\text { implementing photonic quantum computers. }\end{array}$ \\
\hline
\end{tabular}




\subsection{Comparing Common Types of Quantum Bit Technologies}

The search for a suitable physical system in which to implement quantum logic operations has been going on for a long time. In Table 3, we discuss three major qubit technology options for building a quantum computer. These quantum technologies, trapped-ion qubits [24,25], superconducting qubits [26,27], and photonic qubits [28,29], are thought to be well advanced.

Table 3. A comparison of three qubit technologies: trapped ion, superconducting, and photonic qubits is shown in the table based on their operations and fundamental properties.

\begin{tabular}{|c|c|c|}
\hline Trapped Ion Qubit & Superconducting Qubit & Photonic Qubit \\
\hline $\begin{array}{l}\text { To produce qubits, lasers are used to ion- } \\
\text { ize atoms and trap them in electric po- } \\
\text { tentials. The status of the qubits is then } \\
\text { measured using an extra laser. }\end{array}$ & $\begin{array}{l}\text { The qubits are created by combining a } \\
\text { superconducting resonator with a nonlin- } \\
\text { ear inductor to make an artificial atom. }\end{array}$ & $\begin{array}{l}\text { The squeezed state (light working as } \\
\text { qubit) is created by distributing laser light } \\
\text { to an array of squeezers (microscopic de- } \\
\text { vices comprised of relatively small ring } \\
\text { resonators). }\end{array}$ \\
\hline $\begin{array}{l}\text { Stable qubits can be generated using } \\
\text { trapped ion technology, and forming an } \\
\text { entangled state is simple. Working with } \\
\text { large numbers of qubits in this system is } \\
\text { challenging, and implementing a whole } \\
\text { quantum algorithm is even more compli- } \\
\text { cated. Decoherence is a difficult problem } \\
\text { to solve. }\end{array}$ & $\begin{array}{l}\text { Building and accurately measuring } \\
\text { qubits with superconducting technology } \\
\text { is simple. These qubits have a nanosec- } \\
\text { ond time scale and a quick decoherence } \\
\text { time. Qubits must be cooled to near ab- } \\
\text { solute zero to function, and computation } \\
\text { is subject to quantum noise. }\end{array}$ & $\begin{array}{l}\text { Qubits are far more stable in photonic } \\
\text { technology and can readily entangle a } \\
\text { huge number of photons. It is possible } \\
\text { to perform computation at room tempera- } \\
\text { ture, but it is less fault-tolerant, and er- } \\
\text { ror correction is harder. According to } \\
\text { this technique, quantum supremacy is at- } \\
\text { tained. }\end{array}$ \\
\hline
\end{tabular}

\subsection{Challenges in the Field of Quantum Computation}

As we get closer to a fully scalable quantum computing age, the current state of quantum computation faces many hurdles, from experimental to theoretical. Professionals in the field have recognized a few issues, which we summarize here.

\subsubsection{Experimental Challenges of Quantum Computing: Quantum Computers}

Neutral atoms for quantum computing by [30] stated that although quantum computers have been shown to outperform traditional computers, there is yet to be a quantum computer that can perform an operation that cannot be emulated on a regular computer. Because a quantum computer takes around 50 qubits and several gates ranging from $10^{4}$ to achieve a calculation that cannot be emulated on a conventional computer, this is essentially a scale-up problem. In the concluding section of their article, David et al. stated that: "From the view of an experimental physicist, the task of exerting precise control over a large number of individual quantum particles is a grand challenge" [30]. They went further to explain that the following requirements must be met for successful quantum computation:

- $\quad$ Putting atoms in precise quantum states;

- Manipulating the interactions of the atoms to carry out logical procedures;

- $\quad$ Obtaining the computational output by monitoring the resultant states.

\subsubsection{Theoretical Challenges of Quantum Computing: Quantum Simulators}

Quantum machine learning by Biamonte et al. [19] noted that quantum algorithms could only outperform traditional algorithms in exceptional circumstances, according to research. The quantum speedup, which is the outcome of superimposing a quantum state, was the most popular at the time. They thoroughly described the concept of quantum speedup. After their research, four fascinating questions were posed. These questions impacted research focus, which is still happening today since it would provide a more concise way to apply quantum machine learning to everyday applications-the input, output, costing and benchmarking issues were the topics of discussion.

Because quantum algorithms have been shown to be faster than classical methods, data encoding takes up more time (converting classical data to quantum data since the 
readily available data are classical). Until a more rapid encoding program is produced, this is a significant disadvantage. In this domain, several efforts have been made to solve the input problem. In [31], a demonstration of how data can be mapped into a particular space where quantum states exist was exploited. In the work of Schuld [32], it was pointed out that machine learning and quantum computing have certain similarities. This resemblance is known as the "kernel model." In simple terms, a kernel model is a type of machine learning that characterizes information (data) based on how similar and distinct they are from one another. A clear picture of data encoding (embedding) was offered in a more recent publication by Schuld [33]. This article did not directly address the cost of data encoding, but it did provide a concise overview of the various data encoding methods.

\section{Quantum Computing's Chronology and Origin Sequence}

In this part, we give a quantum chronology that stretches from the 1935 EPR paradox [34] to the year 2020. A diagram demonstrating the flow of quantum computing processes related to the three science domains mentioned earlier is also included.

\subsection{Quantum Computing Chronology}

Since the 1935 observation of the EPR paradox, various research projects have resulted in the development of quantum computing systems and cutting-edge technologies. We have put together a timeline in Figure 8 and Table 4 showing how these changes have proceeded over time.

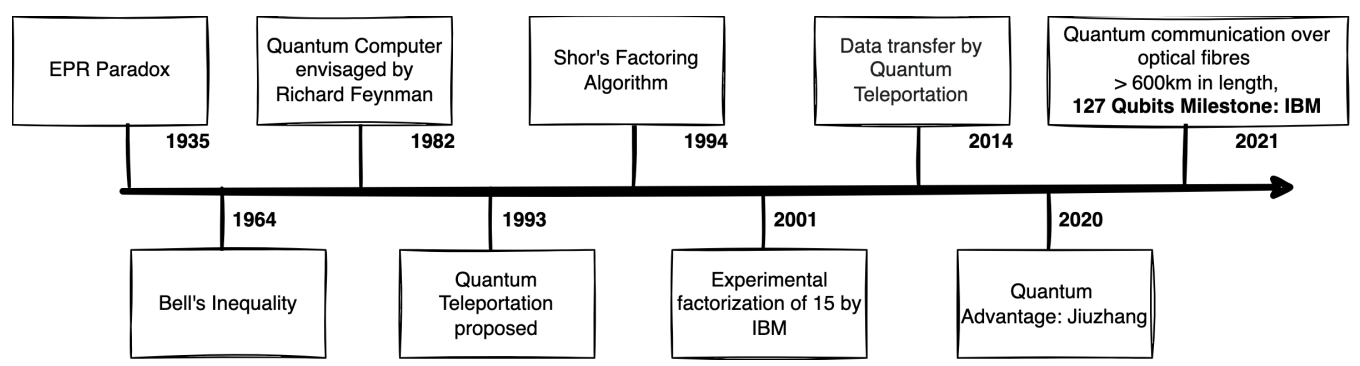

Figure 8. A short timeline exploring the development of quantum technology dating back to 1935.

Table 4. From 1935 until the present, a brief history of quantum technology development.

\begin{tabular}{lll}
\hline Year & Activity & Reference \\
\hline 1935 & EPR Paradox & {$[34]$} \\
1964 & Bell's Inequality & {$[35]$} \\
1982 & Quantum Computer envisaged by Richard Feynman & {$[36]$} \\
1993 & Quantum Teleportation proposed & {$[37]$} \\
1994 & Shor's Factoring Algorithm & {$[38]$} \\
2001 & Experimental factorization of 15 by IBM & {$[39]$} \\
2014 & Data transfer by Quantum Teleportation & {$[40]$} \\
2020 & Quantum Advantage: Jiuzhang & {$[41]$} \\
2021 & Quantum communication over optical fibers over 600 km in length & {$[42]$} \\
2021 & 127 Qubits Milestone: IBM & {$[43]$} \\
\hline
\end{tabular}

\subsection{From Physics to Quantum Computing}

The origins of quantum computing may be traced back to two disciplines of physics: modern and classical. The convergence and divergence of the quantum computing discipline are seen in Figure 9. In this context, some contributions and applications on high energy physics have been reported [44-47]. 


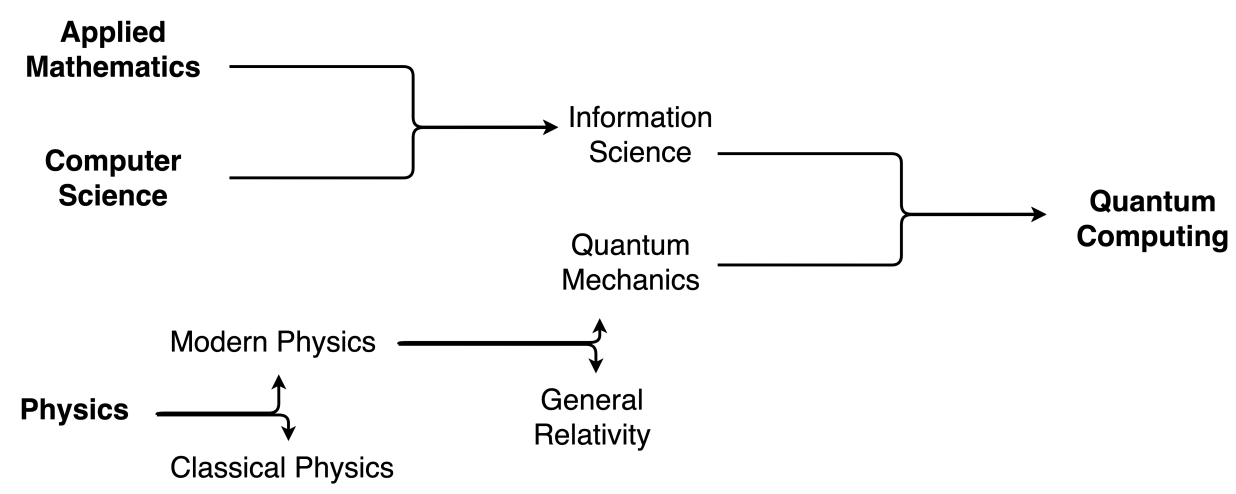

Figure 9. Quantum computing journey from two fields of science: information science and quantum physics.

\section{Discussion}

\subsection{State of the Art}

Colin Williams published the first book on quantum computing, dubbed Explorations in Quantum Computing [48]. However, significant advancements in the subject have occurred since the book's release in 1998. We investigated the progress of quantum information science as it applies to three essential disciplines: quantum computing, quantum sensing, and quantum communication, all of which were discussed previously. We executed a series of searches on the well-known Scopus database (for no apparent reason) to generate data on publications published on topics closely connected to these fields. Quantum sensing, quantum metrology, quantum sensor, quantum hypothesis testing, and/or quantum radar were some of the themes we considered for "quantum sensing and metrology". For "quantum computing and algorithms", we considered topics such as quantum computing, quantum computer, quantum computation, and/or quantum algorithm. Similarly, quantum communication, quantum network, quantum cryptography, quantum modem, quantum internet, and/or quantum bus were the selected topics for "quantum communication". The time frame for the search was 1996 through 2020 (over 20 years of its existence). The following is the search query, data, and visual plot shown in Figures 10 and 11.

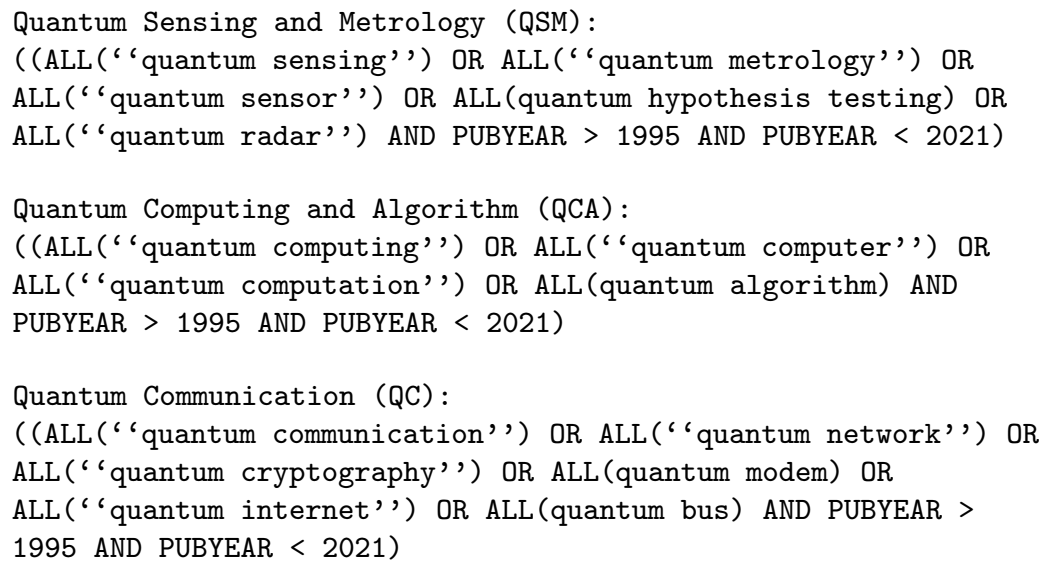

Figure 10. Query search used in Scopus database to generate data of the publications on the topics, quantum sensing, quantum communication, and quantum computing from 1996 to 2020. 


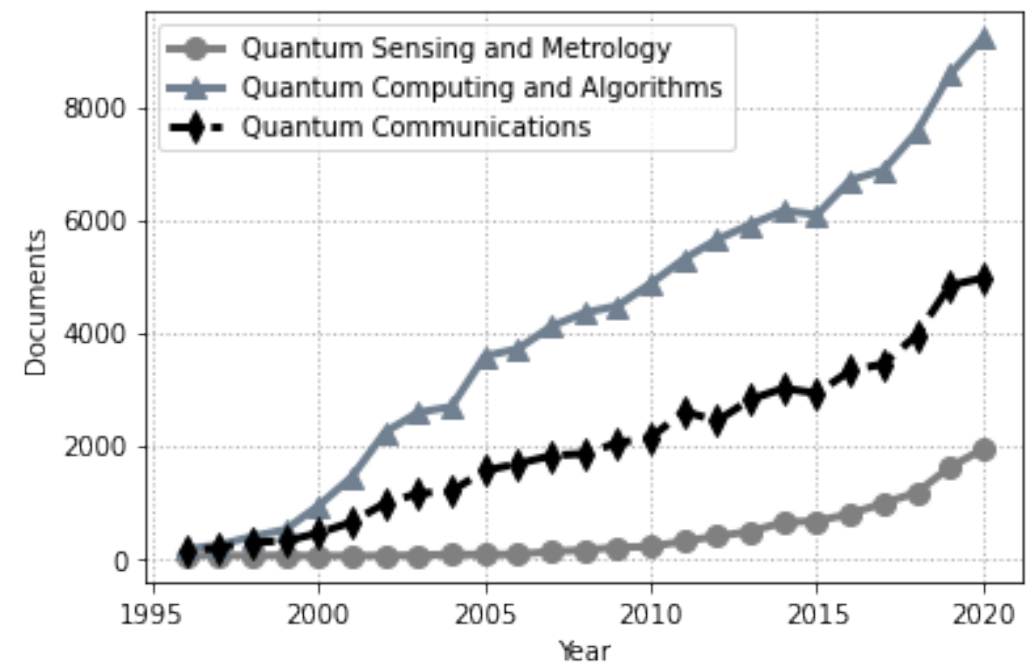

Figure 11. Quantum information science progress report. This graph shows the progression of the number of published documents in the areas of quantum sensing and metrology, quantum computing and algorithms, and quantum communications, respectively. It dates from 1995 through 2020.

In the future, we may be confronted with the possibility of using a quantum computer for everyday tasks. Quantum computing, on the other hand, has yet to achieve that aim, and as a result, it is frequently referred to as "near-term" computing.

Quantum computing is a subset of artificial intelligence that combines quantum mechanics concepts with linear algebra and computer science functions to create a supercomputer. It faces several challenges as a developing field. These difficulties exist in both the hardware and software aspects of the sector. Despite this, there are positive advances that push us in the path of one day realizing the potential it has, hopefully in our lifetime.

\subsection{Emerging Quantum Machine Learning Technology}

Traditional machine learning's effective problem-solving application has shown limitations in areas such as the difficulty of searching algorithms [49] and the costly estimation of kernel functions due to vast feature spaces, to name a few. Theoretical answers to some of them and others were established in QML's recent advances. A good example can be found in the work of [31], where they experimentally achieved a quantum feature space-based classifier. A number of quantum classifiers have been implemented recently, as reported in [50], and their performance is frequently compared to that of their classical counterparts. There have also been advancements in the field of quantum deep learning [51], with recent work by [52] implementing a quantum generative adversarial network on a superconducting quantum processor for learning and the generation of real-world handwritten digital images, and [53] implementing quantum convolutional neural networks (QCNN) for different applications; among them is the use of QCNN to create a quantum error correction method that is optimized for a particular error model. It is vital to highlight the combinatory "hybrid quantum-classical algorithms", which include recent advances, such as those seen in [54], where hybrid quantum variational autoencoder was applied to a representation learning task as well as the work of [55] implementing hybrid quantum-classical convolutional neural network on a Tetris dataset for classification.

\section{Conclusions}

The study of quantum computational learning theory encompasses a wide range of topics. An extensive survey article that covers every aspect of the field, we believe, would be overwhelming. However, in a reasonable span, this succinct review presents a diverse range of topics, all of which are made more accessible using charts, tables, and figures. As a result, we provide a suitable first reference to modern quantum computing for a wider audience with no prior knowledge of the area. Since we recognize that our introductory 
review will lead to more specific questions, Table 5 lists resources for future reading, aiming to provide an in-depth grasp of the quantum computing field.

Table 5. A collection of further reading resources encompassing subfields, such as quantum machine learning, quantum algorithms, and quantum ethics, is provided below.

\begin{tabular}{ll}
\hline Interesting Reads & Reference \\
\hline Quantum Learning and Optimization & {$[56-59]$} \\
Quantum Machine Learning & {$[19,32,33,44,54,60]$} \\
Quantum Fault Tolerance and Error Correction & {$[61-65]$} \\
Quantum System Simulation & {$[21,25,66-69]$} \\
Quantum Algorithm Designs & {$[70-75]$} \\
Quantum Hardware Test Development & {$[76-80]$} \\
Quantum Cryptography & {$[81-87]$} \\
Hybrid (Quantum-Classical) Computing & {$[54,55,88-90]$} \\
Ethical Quantum Computing & {$[91-93]$} \\
\hline
\end{tabular}

Finally, we assert that the relationship between classical artificial intelligence, machine learning, and deep learning are in direct dependency on quantum artificial intelligence, which includes quantum computation systems in their entirety, quantum machine learning, which includes quantum Fourier transforms, amplitude amplification, algorithms, and quantum deep learning, which includes quantum neural networks, quantum convolutional neural networks, and other concepts. Figure 12 depicts the symbiotic dependencies between the aforementioned knowledge areas.

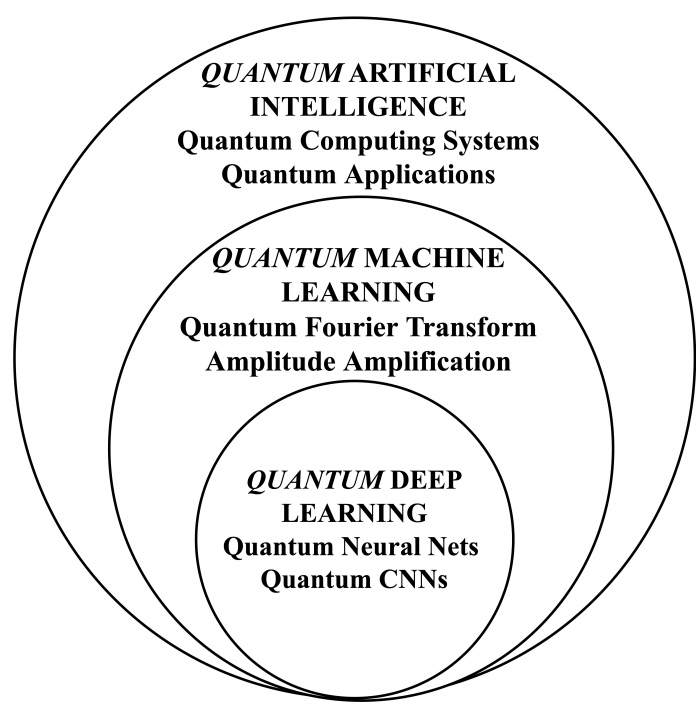

Figure 12. Quantum artificial intelligence is a superset of quantum machine learning and quantum deep learning.

Author Contributions: Conceptualization, O.A., J.O. and P.R.; methodology, O.A. and P.R.; formal analysis, J.O.; investigation, O.A.; writing-original draft preparation, O.A.; writing-review and editing, P.R.; visualization, J.O. and O.A.; supervision, P.R.; funding acquisition, P.R. All authors have read and agreed to the published version of the manuscript.

Funding: This material is based upon work supported by the National Science Foundation under Grant CHE-1905043 and CNS-2136961.

Institutional Review Board Statement: Not applicable.

Informed Consent Statement: Not applicable.

Data Availability Statement: No new data were created or analyzed in this study. Data sharing is not applicable to this article. 
Acknowledgments: The work presented here was supported in part by the Baylor AI lab in Baylor University's Department of Computer Science.

Conflicts of Interest: The authors declare no conflict of interest.

\section{References}

1. Schuld, M.; Petruccione, F. Supervised Learning with Quantum Computers; Springer: Berlin/Heidelberg, Germany, 2018 ; Volume 17.

2. Montanaro, A. Quantum algorithms: An overview. Npj Quantum Inf. 2016, 2, 15023. [CrossRef]

3. Jordan, S. The Quantum Algorithm Zoo. 2021. Available online: http://math.nist.gov/quantum/zoo/ (accessed on 1 November 2021).

4. Marais, A.; Adams, B.; Ringsmuth, A.K.; Ferretti, M.; Gruber, J.M.; Hendrikx, R.; Schuld, M.; Smith, S.L.; Sinayskiy, I.; Krüger, T.P.; et al. The future of quantum biology. J. R. Soc. Interface 2018, 15, 20180640. [CrossRef]

5. Biamonte, J.; Faccin, M.; De Domenico, M. Complex networks from classical to quantum. Commun. Phys. 2019, 2, 53. [CrossRef]

6. McMahon, D. Quantum Mechanics Demystified; McGraw-Hill Education: New York, NY, USA, 2013.

7. Samuel, A.L. Some studies in machine learning using the game of checkers. IBM J. Res. Dev. 1959, 3, 210-229. [CrossRef]

8. Alzubi, J.; Nayyar, A.; Kumar, A. Machine Learning from Theory to Algorithms: An Overview. J. Phys. Conf. Ser. 2018, 1142, 012012. [CrossRef]

9. $\quad$ Rivas, P. Deep Learning for Beginners: A Beginner's Guide to Getting Up and Running with Deep Learning from Scratch Using Python; Packt Publishing Ltd.: Birmingham, UK, 2020.

10. Mehta, P.; Bukov, M.; Wang, C.H.; Day, A.G.; Richardson, C.; Fisher, C.K.; Schwab, D.J. A high-bias, low-variance introduction to machine learning for physicists. Phys. Rep. 2019, 810,1-124. [CrossRef]

11. Mahesh, B. Machine Learning Algorithms-A Review. Int. J. Sci. Res. (IJSR) 2020, 9, 381-386.

12. Bonaccorso, G. Machine Learning Algorithms; Packt Publishing Ltd.: Birmingham, UK, 2017.

13. Wittek, P. Quantum Machine Learning: What Quantum Computing Means to Data Mining; Academic Press: Cambridge, MA, USA, 2014.

14. Nielsen, M.A.; Chuang, I.L. Quantum computation and quantum information. Phys. Today 2001, 54, 60.

15. McMahon, D. Quantum Computing Explained; John Wiley \& Sons: Hoboken, NJ, USA, 2007.

16. Mermin, N.D. Quantum Computer Science: An Introduction; Cambridge University Press: Cambridge, UK, 2007.

17. Kaye, P.; Laflamme, R.; Mosca, M. An Introduction to Quantum Computing; Oxford University Press on Demand: Oxford, UK, 2007.

18. Grumbling, E.; Horowitz, M. Adiabatic Quantum Computing and Quantum Annealing. In Quantum Computing: Progress and Prospects; The National Academies Press: Washington DC, USA, 2019. [CrossRef]

19. Biamonte, J.; Wittek, P.; Pancotti, N.; Rebentrost, P.; Wiebe, N.; Lloyd, S. Quantum machine learning. Nature 2017, 549, 195-202. [CrossRef]

20. Grant, E.K.; Humble, T.S. Adiabatic Quantum Computing and Quantum Annealing; Oxford University Press: Oxford, UK, 2020. [CrossRef]

21. Georgescu, I.M.; Ashhab, S.; Nori, F. Quantum simulation. Rev. Mod. Phys. 2014, 86, 153. [CrossRef]

22. Michielsen, K.; Nocon, M.; Willsch, D.; Jin, F.; Lippert, T.; De Raedt, H. Benchmarking gate-based quantum computers. Comput. Phys. Commun. 2017, 220, 44-55. [CrossRef]

23. Mosca, M. Quantum algorithms. arXiv 2008, arXiv:0808.0369.

24. Bruzewicz, C.D.; Chiaverini, J.; McConnell, R.; Sage, J.M. Trapped-ion quantum computing: Progress and challenges. Appl. Phys. Rev. 2019, 6, 021314. [CrossRef]

25. Blatt, R.; Roos, C.F. Quantum simulations with trapped ions. Nat. Phys. 2012, 8, 277-284. [CrossRef]

26. Krantz, P.; Kjaergaard, M.; Yan, F.; Orlando, T.P.; Gustavsson, S.; Oliver, W.D. A quantum engineer's guide to superconducting qubits. Appl. Phys. Rev. 2019, 6, 021318. [CrossRef]

27. Huang, H.L.; Wu, D.; Fan, D.; Zhu, X. Superconducting quantum computing: A review. Sci. China Inf. Sci. 2020, 63, 1-32. [CrossRef]

28. Kok, P.; Munro, W.J.; Nemoto, K.; Ralph, T.C.; Dowling, J.P.; Milburn, G.J. Linear optical quantum computing with photonic qubits. Rev. Mod. Phys. 2007, 79, 135. [CrossRef]

29. Nicolas, A.; Veissier, L.; Giner, L.; Giacobino, E.; Maxein, D.; Laurat, J. A quantum memory for orbital angular momentum photonic qubits. Nat. Photonics 2014, 8, 234-238. [CrossRef]

30. Weiss, D.S.; Saffman, M. Quantum computing with neutral atoms. Phys. Today 2017, 70, 7, 44. [CrossRef]

31. Havlíček, V.; Córcoles, A.D.; Temme, K.; Harrow, A.W.; Kandala, A.; Chow, J.M.; Gambetta, J.M. Supervised learning with quantum-enhanced feature spaces. Nature 2019, 567, 209-212. [CrossRef]

32. Schuld, M. Machine Learning in Quantum Spaces; Nature Publishing Group: Berlin, Germany, 2019.

33. Schuld, M. Quantum machine learning models are kernel methods. arXiv 2021, arXiv:2101.11020.

34. Einstein, A.; Podolsky, B.; Rosen, N. Can quantum-mechanical description of physical reality be considered complete? Phys. Rev. 1935, 47, 777. [CrossRef]

35. Bell, J.S. On the einstein podolsky rosen paradox. Phys. Phys. Fiz. 1964, 1, 195. [CrossRef] 
36. Feynman, R.P. Simulating physics with computers. In Feynman and Computation; CRC Press: Boca Raton, FL, USA, 2018; pp. 133-153.

37. Bennett, C.H.; Brassard, G.; Crépeau, C.; Jozsa, R.; Peres, A.; Wootters, W.K. Teleporting an unknown quantum state via dual classical and Einstein-Podolsky-Rosen channels. Phys. Rev. Lett. 1993, 70, 1895. [CrossRef]

38. Shor, P.W. Algorithms for quantum computation: Discrete logarithms and factoring. In Proceedings of the 35th Annual Symposium on Foundations of Computer Science, Santa Fe, NM, USA, 20-22 November 1994; pp. 124-134.

39. Vandersypen, L.M.; Steffen, M.; Breyta, G.; Yannoni, C.S.; Sherwood, M.H.; Chuang, I.L. Experimental realization of Shor's quantum factoring algorithm using nuclear magnetic resonance. Nature 2001, 414, 883-887. [CrossRef]

40. Pfaff, W.; Hensen, B.J.; Bernien, H.; van Dam, S.B.; Blok, M.S.; Taminiau, T.H.; Tiggelman, M.J.; Schouten, R.N.; Markham, M.; Twitchen, D.J.; et al. Unconditional quantum teleportation between distant solid-state quantum bits. Science 2014, 345, 532-535. [CrossRef]

41. Zhong, H.S.; Wang, H.; Deng, Y.H.; Chen, M.C.; Peng, L.C.; Luo, Y.H.; Qin, J.; Wu, D.; Ding, X.; Hu, Y.; et al. Quantum computational advantage using photons. Science 2020, 370, 1460-1463. [CrossRef]

42. Daiss, S.; Langenfeld, S.; Welte, S.; Distante, E.; Thomas, P.; Hartung, L.; Morin, O.; Rempe, G. A quantum-logic gate between distant quantum-network modules. Science 2021, 371, 614-617. [CrossRef]

43. Ball, P. First quantum computer to pack 100 qubits enters crowded race. Nature 2021, 599, 542. [CrossRef]

44. Guan, W.; Perdue, G.; Pesah, A.; Schuld, M.; Terashi, K.; Vallecorsa, S.; Vlimant, J.R. Quantum machine learning in high energy physics. Mach. Learn. Sci. Technol. 2021, 2, 011003. [CrossRef]

45. Vijayasri, I.; Javier, O.-D. Quantum Machine Learning Concepts and Applications. 2020. Available online: https://research latinxinai.org/workshops/neurips/neurips-2020.html (accessed on 1 November 2021).

46. Orduz-Ducuara, J.A. Quantum Machine Learning and Higgs Phenomenology. 2020, in preparation.

47. Orduz, J.; Iyer, V. Quantum Machine Learning concepts for Physicists. Tecnología Educativa, Revista CONAIC, Mexico City, Mexico, 2021; Volume VIII, pp. 71-75. Número 2, Mayo-Agosto 2021. ISSN 2395-9061. Available online: https://terc.mx/index. $\mathrm{php} /$ terc (accessed on 1 November 2021).

48. Williams, C.P. Explorations in Quantum Computing; Springer Science \& Business Media: Berlin/Heidelberg, Germany, 2010.

49. Khanal, B.; Rivas, P.; Orduz, J. Quantum Machine Learning: A Case Study of Grover's Algorithm. In Proceedings of the 19th International Conference on Scientific Computing (CSC 2021), Las Vegas, NV, USA, 15-17 December 2021.

50. Jui, T.; Ayoade, O.; Rivas, P.; Orduz, J. Performance Analysis of Quantum Machine Learning Classifiers. In Proceedings of the NeurIPS 2021 Workshop LatinX in AI, Virtual Event, 7 December 2021.

51. Garg, S.; Ramakrishnan, G. Advances in quantum deep learning: An overview. arXiv 2020, arXiv:2005.04316.

52. Huang, H.L.; Du, Y.; Gong, M.; Zhao, Y.; Wu, Y.; Wang, C.; Li, S.; Liang, F.; Lin, J.; Xu, Y.; et al. Experimental quantum generative adversarial networks for image generation. Phys. Rev. Appl. 2021, 16, 024051. [CrossRef]

53. Cong, I.; Choi, S.; Lukin, M.D. Quantum convolutional neural networks. Nat. Phys. 2019, 15, 1273-1278. [CrossRef]

54. Rivas, P.; Zhao, L.; Orduz, J. Hybrid Quantum Variational Autoencoders for Representation Learning. In Proceedings of the 19th International Conference on Scientific Computing (CSC 2021), Las Vegas, NV, USA, 15-17 December 2021.

55. Liu, J.; Lim, K.H.; Wood, K.L.; Huang, W.; Guo, C.; Huang, H.L. Hybrid quantum-classical convolutional neural networks. Sci. China Phys. Mech. Astron. 2021, 64, 290311. [CrossRef]

56. Arunachalam, S.; de Wolf, R. Guest column: A survey of quantum learning theory. ACM Sigact News 2017, 48, 41-67. [CrossRef]

57. Hogg, T.; Portnov, D. Quantum optimization. Inf. Sci. 2000, 128, 181-197. [CrossRef]

58. Li, Y.; Tian, M.; Liu, G.; Peng, C.; Jiao, L. Quantum optimization and quantum learning: A survey. IEEE Access 2020, 8, $23568-23593$. [CrossRef]

59. Harrigan, M.P.; Sung, K.J.; Neeley, M.; Satzinger, K.J.; Arute, F.; Arya, K.; Atalaya, J.; Bardin, J.C.; Barends, R.; Boixo, S.; et al. Quantum approximate optimization of non-planar graph problems on a planar superconducting processor. Nat. Phys. 2021, 17, 332-336. [CrossRef]

60. Schuld, M.; Sinayskiy, I.; Petruccione, F. An introduction to quantum machine learning. Contemp. Phys. 2015, 56, 172-185. [CrossRef]

61. Cross, A.W.; DiVincenzo, D.P.; Terhal, B.M. A comparative code study for quantum fault-tolerance. arXiv 2007, arXiv:0711.1556

62. Schumacher, B.; Nielsen, M.A. Quantum data processing and error correction. Phys. Rev. A 1996, 54, 2629. [CrossRef] [PubMed]

63. Gottesman, D. Theory of fault-tolerant quantum computation. Phys. Rev. A 1998, 57, 127. [CrossRef]

64. Knill, E.; Laflamme, R.; Viola, L. Theory of quantum error correction for general noise. Phys. Rev. Lett. 2000, 84, 2525. [CrossRef]

65. Devitt, S.J.; Munro, W.J.; Nemoto, K. Quantum error correction for beginners. Rep. Prog. Phys. 2013, 76, 076001. [CrossRef]

66. Lloyd, S. Universal quantum simulators. Science 1996, 273, 1073-1078. [CrossRef]

67. Barreiro, J.T.; Müller, M.; Schindler, P.; Nigg, D.; Monz, T.; Chwalla, M.; Hennrich, M.; Roos, C.F.; Zoller, P.; Blatt, R. An open-system quantum simulator with trapped ions. Nature 2011, 470, 486-491. [CrossRef]

68. Aspuru-Guzik, A.; Walther, P. Photonic quantum simulators. Nat. Phys. 2012, 8, 285-291. [CrossRef]

69. Kim, K.; Chang, M.S.; Korenblit, S.; Islam, R.; Edwards, E.E.; Freericks, J.K.; Lin, G.D.; Duan, L.M.; Monroe, C. Quantum simulation of frustrated Ising spins with trapped ions. Nature 2010, 465, 590-593. [CrossRef]

70. Shao, C.; Li, Y.; Li, H. Quantum algorithm design: Techniques and applications. J. Syst. Sci. Complex. 2019, 32, 375-452. [CrossRef]

71. Behrman, E.C.; Steck, J.E.; Kumar, P.; Walsh, K. Quantum algorithm design using dynamic learning. arXiv 2008, arXiv:0808.1558 
72. Cafaro, C. Geometric algebra and information geometry for quantum computational software. Phys. A Stat. Mech. Appl. 2017, 470, 154-196. [CrossRef]

73. Bang, J.; Ryu, J.; Yoo, S.; Pawłowski, M.; Lee, J. A strategy for quantum algorithm design assisted by machine learning. New J. Phys. 2014, 16, 073017. [CrossRef]

74. Lin, J.; Lai, Z.Y.; Li, X. Quantum adiabatic algorithm design using reinforcement learning. Phys. Rev. A 2020, $101,052327$. [CrossRef]

75. Gushanskiy, S.; Polenov, M.; Potapov, V. The methodology of implementation and simulation of quantum algorithms and processes. In Proceedings of the 2017 IEEE 11th International Conference on Application of Information and Communication Technologies (AICT), Moscow, Russia, 20-22 September 2017; pp. 1-5.

76. Vinci, W.; Albash, T.; Paz-Silva, G.; Hen, I.; Lidar, D.A. Quantum annealing correction with minor embedding. Phys. Rev. A 2015, 92, 042310. [CrossRef]

77. Tremsin, A.S.; Siegmund, O.H.; Vallerga, J.V.; Hull, J.S.; Abiad, R. Cross-strip readouts for photon counting detectors with high spatial and temporal resolution. IEEE Trans. Nucl. Sci. 2004, 51, 1707-1711. [CrossRef]

78. Chiesa, A.; Tacchino, F.; Grossi, M.; Santini, P.; Tavernelli, I.; Gerace, D.; Carretta, S. Quantum hardware simulating fourdimensional inelastic neutron scattering. Nat. Phys. 2019, 15, 455-459. [CrossRef]

79. Mykhailovaa, M.; Soekena, M. Testing Quantum Programs using Q\# and Microsoft Quantum Development Kit. In Proceedings of the 2nd Quantum Software and Engineering Workshop (QSET'21), Virtual, 19 October 2021.

80. Wootton, J.R. Procedural generation using quantum computation. In Proceedings of the International Conference on the Foundations of Digital Games, Bugibba, Malta, 15-18 September 2020; pp. 1-8.

81. Ekert, A.K. Quantum cryptography based on Bell's theorem. Phys. Rev. Lett. 1991, 67, 661. [CrossRef]

82. Gisin, N.; Ribordy, G.; Tittel, W.; Zbinden, H. Quantum cryptography. Rev. Mod. Phys. 2002, 74, 145. [CrossRef]

83. Horodecki, R.; Horodecki, P.; Horodecki, M.; Horodecki, K. Quantum entanglement. Rev. Mod. Phys. 2009, 81, 865. [CrossRef]

84. Scarani, V.; Bechmann-Pasquinucci, H.; Cerf, N.J.; Dušek, M.; Lütkenhaus, N.; Peev, M. The security of practical quantum key distribution. Rev. Mod. Phys. 2009, 81, 1301. [CrossRef]

85. Bennett, C.H.; Brassard, G.; Mermin, N.D. Quantum cryptography without Bell's theorem. Phys. Rev. Lett. 1992, 68, 557. [CrossRef] [PubMed]

86. Bennett, C.H.; Bessette, F.; Brassard, G.; Salvail, L.; Smolin, J. Experimental quantum cryptography. J. Cryptol. 1992, 5, 3-28. [CrossRef]

87. Gisin, N.; Thew, R. Quantum communication. Nat. Photonics 2007, 1, 165-171. [CrossRef]

88. McCaskey, A.; Dumitrescu, E.; Chen, M.; Lyakh, D.; Humble, T. Validating quantum-classical programming models with tensor network simulations. PLoS ONE 2018, 13, e0206704. [CrossRef]

89. Lanzagorta, M.; Uhlmann, J.K. Hybrid quantum-classical computing with applications to computer graphics. In Proceedings of the ACM SIGGRAPH 2005 Courses, Los Angeles, CA, USA, 31 July-4 August 2005.

90. Bergholm, V.; Izaac, J.; Schuld, M.; Gogolin, C.; Alam, M.S.; Ahmed, S.; Arrazola, J.M.; Blank, C.; Delgado, A.; Jahangiri, S.; et al. Pennylane: Automatic differentiation of hybrid quantum-classical computations. arXiv 2018, arXiv:1811.04968.

91. Kop, M. Establishing a Legal-Ethical Framework for Quantum Technology. Yale Law Sch. Yale J. Law Technol. (YJoLT) Rec. 2 March 2021. Available online: https:/ / ssrn.com/abstract=3814422 (accessed on 1 November 2021).

92. Perrier, E. Ethical Quantum Computing: A Roadmap. arXiv 2021, arXiv:2102.00759.

93. Perrier, E. Quantum Fair Machine Learning. arXiv 2021, arXiv:2102.00753. 